\title{
Case report: the first case of human infection by adult of SPIROMETRA ERINACEIEUROPAEI in VIETNAM
}

\author{
Anh Tran Le $1^{*}$, Le-Quyen Thi Do ${ }^{2}$, Huong-Binh Thi Nguyen ${ }^{3}$, Hong-Ngoc Thi Nguyen ${ }^{4}$ and Anh Ngoc Do ${ }^{5}$
}

\begin{abstract}
Background: Tapeworms of the genus Spirometra include species whose larval stages can infect humans, causing a disease called sparganosis. Cases of human infection with adult worms are very rare and have been reported in Korea and China. Here we report the first case of human infection with an adult of Spirometra erinaceieuropaei in Vietnam.

Case presentation: A 23-year-old male was admitted to 103 Military Hospital, Hanoi, Vietnam with fever, weight loss and epigastric discomfort. Preliminary diagnosis based on discovery of parasite eggs in his faeces incorrectly determined a fluke as the agent of the infection and praziquantel was prescribed. Two days later he passed out proglottids in his stool. The tapeworm was identified as Spirometra erinaceieuropaei using morphological and molecular tools.
\end{abstract}

Conclusion: This is the first case of human infection with adult worm of Spirometra erinaceieuropaei in Vietnam.

Keywords: Case report, Spirometraerinacei europaei, Adult worm, Human, Vietnam, Molecular analysis

\section{Background}

Spirometra is a tapeworm genus in the order Diphyllobothriidea [1] that includes several species: S. erinacei (=S. erinaceieuropaei), Sparganum mansoni, Spirometra mansonoides and an aberrant form of Spirometra proliferum [2].

Spirometra has a complicated life cycle with three hosts. The definitive hosts are dogs, cats, and some other mammals where adult worms live in the small intestine and produce unembryonated eggs that are discharged in faeces. Once in fresh water, the unembryonated eggs hatch to become coracidia. The first intermediate hosts are copepods (Cyclops) taking up the coracidia, which develop into procercoid larvae in Cyclops. When fish, reptiles, or amphibians consume the copepods, they become the second intermediate host of Spirometra. Procercoid larvae penetrate the intestinal tract of the second intermediate host, become plerocercoid larvae (sparganum larvae), then migrate to the

\footnotetext{
*Correspondence: anh_It@vmmu.edu.vn

${ }^{1}$ Department of Parasitology, Vietnam Military Medical University (VMMU),

Phung Hung Street, Ha Dong Town, Hanoi, Vietnam

Full list of author information is available at the end of the article
}

subcutaneous tissues and muscles. The cycle begins again as a definitive host takes up a second intermediate host. Many amphibians, reptiles and even mammals can become paratenic hosts when they are infected with spargana [3].

Humans can be accidental definitive, second intermediate or paratenic hosts as well. Larval stages of some species such as S. erinaceieuropaei, S. mansoni, S. mansonoides, S. proliferum can infect humans and cause a disease called sparganosis [4] which is endemic in Asian countries including China [5], Hong Kong [6], South Korea [7], Japan [8], and Thailand [9]. Cases of human infection with adult worms are very rare and have been reported in Korea and China [10, 11].

We report a case of human infection with adult of S. erinaceieuropaei identified by its morphology and genetic analysis in Vietnam. To our knowledge, this is the first case of adult $S$. erinaceieuropaei recovered from human in Vietnam.

\section{Case presentation}

A 23-year-old male was admitted to 103 Hospital (Ha Dong Town, Hanoi, Vietnam) on September 4th, 2012 
with a sixteen-day history of fever and weight loss. The patient had slight epigastric pain migrating to right lower quadrant of the abdomen but no nausea or vomiting. In the first few days in hospital, his stool was watery but no mucus or blood was seen. Physical examination was normal. Complete blood count and liver function tests were performed and all were in a normal range. His blood was screened for hepatitis A, B and C, malaria, liver and lung flukes, Toxocara spp., Strongyloides spp. as well as cultured for microbacteria and the results were negative. Image studies (abdominal ultrasound and chest ray) were normal. With a sixteen-day history of fever the patient was diagnosed with sepsis and treated with a combination of two antibiotics (cefpirome and levofloxacin). Yet he showed no improvement in fever nor abdominal pain. On September 11th, a stool examination revealed a large quantity of ovoid-shaped eggs in sizes of about $60 \times 40 \mu \mathrm{m}$ (Fig. 1). Preliminary diagnosis of fluke was made and a single dose of praziquantel (1200 mg) was prescribed. On September 15th, he expelled pieces of tapeworm strobila, with off-white proglottids, wider than long (about $0.3 \times 0.8 \mathrm{~cm}$ ) in his stool (Fig. 2). Based on these morphological characteristics, the parasite was assigned to Spirometra sp. $[12,10]$.

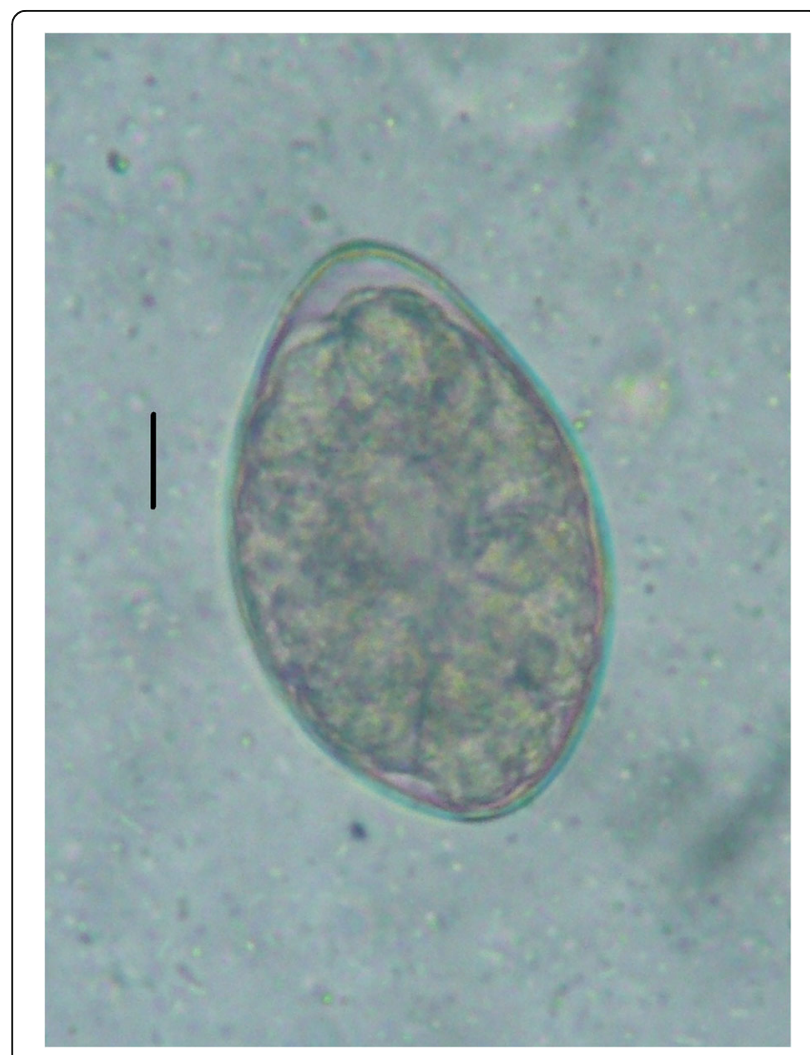

Fig. 1 Ovoid, operculated eggs in stool (bar $=10 \mu \mathrm{m})$
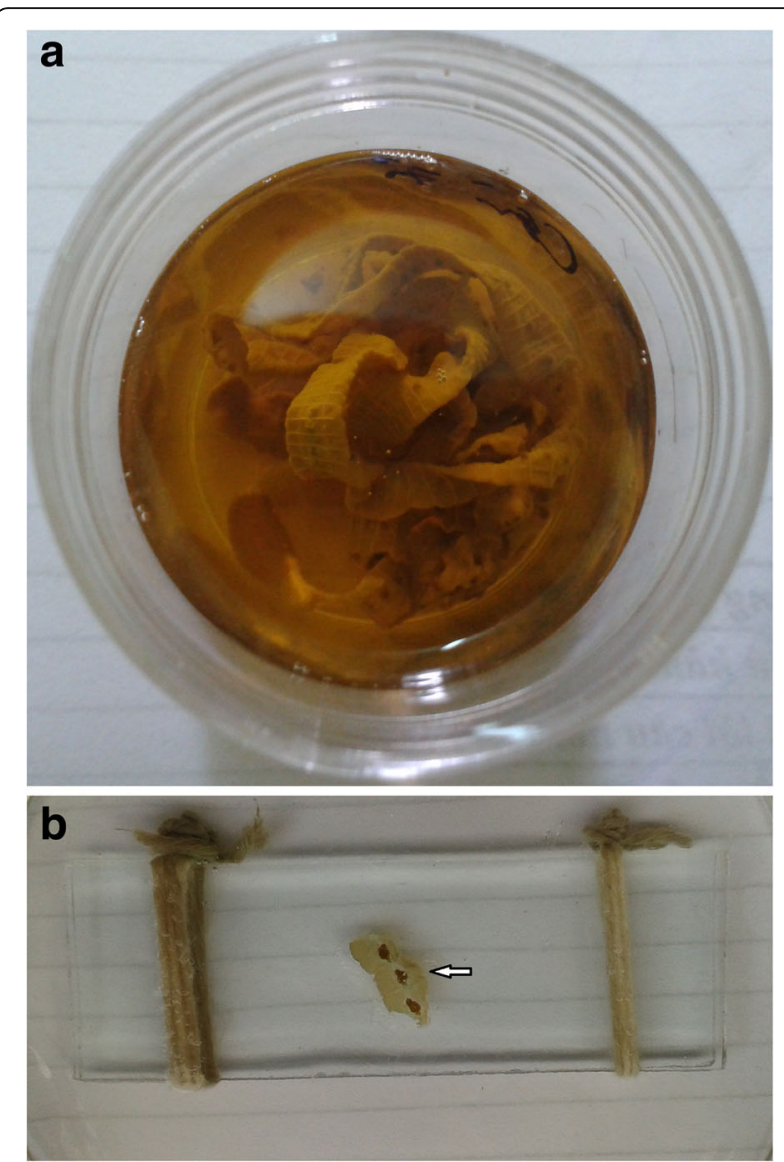

Fig. 2 Wider than long proglottids in the stool after treatment with praziquantel (a: Poglottids in alcohol 70\%. b: Poglottids on a slide (arrow)

Following the treatment of tapeworm, symptoms were quickly resolved. Stool tests for ova and parasite strobila two weeks later were negative. The patient fully recovered and was discharged some days later.

To speciate the worm DNA was extracted from a snip of the worm strobila using QIAamp DNA Micro kit (QIAGEN) according to the manufacturer's instructions. A PCR was performed following to protocol of Hyeong-Kyu Jeon [7]. The $440 \mathrm{bp}$ product of PCR reaction with primers p1f and p1r was sequenced and analyzed by BLAST tool showing 99\% identity with cytochrome c oxidase subunit 1 (Cox 1) gene of $S$. erinaceieuropaei. The sequence was submitted to GenBank (MF682495) Fig. 3.

\section{Discussion}

The most common state of infection with this tapeworm is larvae. Only a few cases of human infection with adult worms have been reported so far $[10,11]$. The patients usually suffered minor health problems and were successfully treated with praziquantel [13]. 


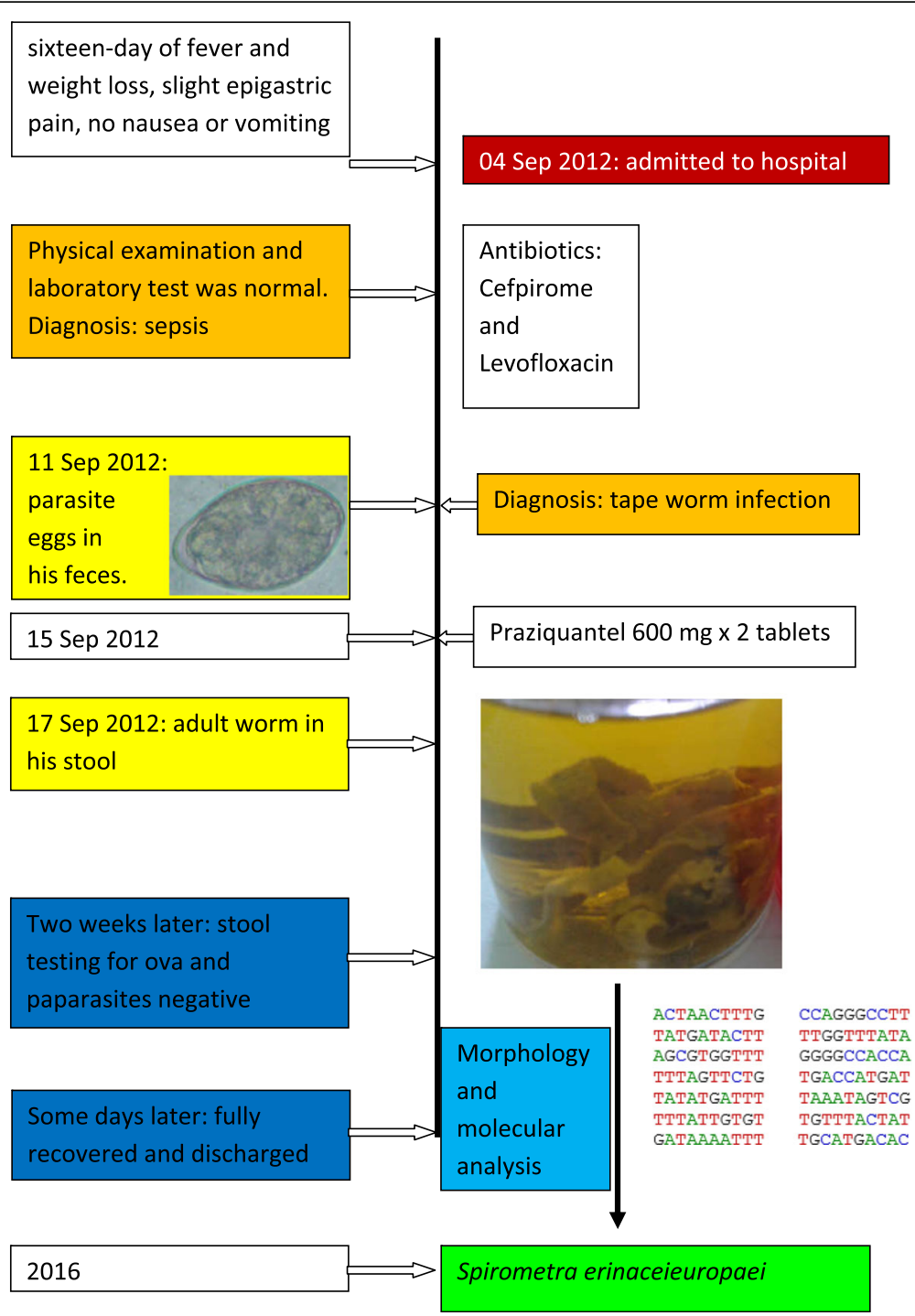

Fig. 3 Timeline

Vietnam and the south-eastern Asia region witness a large number of zoonotic diseases (taeniasis, trichinellosis) relating to the habit of eating raw meat [14]. Vietnamese people also traditionally practice using frog flesh as dressings for open wounds or eyes for medical purposes [15]. Water hygiene is another problem since clean water is not accessible in some areas. These factors lead to Vietnam being a conducive environment for diverse parasitological diseases in general and infections with Spirometra in particular. Although Vietnam is considered an endemic area of sparganosis [12], human clinical cases have been rarely reported, e.g. a subcutaneous sparganosis case published by Vortel el al. [16].

\section{Conclusion}

This is the first case where an adult worm of Spirometra erinaceieuropaei was retrieved from human in Vietnam.
The name of the tapeworm is Spirometra erinaceieuropaei, as confirmed by morphological and molecular tools. Further research to determine the exact epidemiological situation in Vietnam is necessary to facilitate appropriate preventive measures. Health education should be augmented to promote hygienic behaviors such as eating thoroughly cooked meat and drinking boiled water to prevent meat borne and waterborne diseases.

\section{Abbreviations}

bp: Base pairs; Cox1: Cytochrome c oxidase subunit l; DNA: Deoxyribonucleic acid; PCR: Polymerase Chain Reaction

\section{Acknowledgements}

The authors are grateful to Mr. Robert Mayrhofer and Ms. Linh Khanh Le for revising the English text.

Funding

Not applicable. 


\section{Availability of data and materials}

The sequence generated and analysed during the current study is available in the Genbank under the code MF682495.

\section{Authors' contributions}

ATL designed the study and created the final draft of the manuscript. LQDT collected data relating to the Case Report. HBTN, HNTN and AND conducted the study, as well as morphological and molecular analyses. All authors read and approved of the final manuscript.

\section{Ethics approval and consent to participate}

Not applicable.

\section{Consent for publication}

Written informed consent was obtained from the patient for the publicationof this case report and any accompanying images. A copy of the writtenconsent is available for review by the Editor of this journal.

\section{Competing interests}

The authors declare that they have no competing interests.

\section{Publisher's Note}

Springer Nature remains neutral with regard to jurisdictional claims in published maps and institutional affiliations.

\section{Author details}

${ }^{1}$ Department of Parasitology, Vietnam Military Medical University (VMMU) Phung Hung Street, Ha Dong Town, Hanoi, Vietnam. ${ }^{2}$ Department of Infectious Disease, 103 Military Hospital, VMMU, Hanoi, Vietnam. ${ }^{3}$ Department of Molecular Biology, National Institute of Malariology, Parasitology and Entomology (NIMPE), Luong The Vinh Street, Hanoi, Vietnam. ${ }^{4}$ Department of Molecular Biology, National Institute of Malaria, Parasitology and Entomology (NIMPE), Hanoi, Vietnam. ${ }^{5}$ Department of Parasitology, Vietnam Military Medical University (VMMU), Hanoi, Vietnam.

Received: 16 December 2016 Accepted: 3 October 2017

\section{Published online: 10 October 2017}

\section{References}

1. Kuchta R, Brabec J, Scholz T, Bray RA. Suppression of the tapeworm order Pseudophyllidea (Platyhelminthes: Eucestoda) and the proposal of two new orders, Bothriocephalidea and Diphyllobothriidea. Int J Parasitol. 2008;38:49-55.

2. Kuchta R, Scholz T, Brabec J, Narduzzi-Wicht B. Diphyllobothrium, Diplogonoporus, and Spirometra. In: Xiao L, Ryan U, Feng Y, editors. Biology of foodborne parasites. CRC press; 2015. p. 299-326.

3. Zhou X-N, Bergquist R, Olveda R, Utzinger J. Important Helminth infections in Southeast Asia, volume 72: diversity and potential for control and elimination, part a. In: Advances in parasitology. 1 edition. Academic press: 2010.

4. Farrar J, Hotez P, Junghanss T, Kang G, Lalloo D, White NJ. Manson's tropical diseases. 23th Editi. In: Elsevier Saunders; 2013.

5. Li M-W, Song H-Q, Li C, Lin H-Y, Xie W-T, Lin R-Q, et al. Sparganosis in mainland China. Int J Infect Dis [Internet]. 2011 Mar [cited 2014 Dec 16]; 15(3):e154-6. Available from: http://www.ncbi.nlm.nih.gov/pubmed/ 21126898

6. Tang THC, Wong SSY, Lai CKC, Poon RWS, Chan HSY, Wu TC, et al. Molecular identification of Spirometra erinaceieuropaeiTapeworm in cases of human Sparganosis, Hong Kong. Emerg Infect Dis. 2017;23(4):665-668.

7. Jeon HK, Park H, Lee D, Choe S, Kim KH, Huh S, et al. Human infections with spirometra decipiens plerocercoids identified by morphologic and genetic analyses in Korea. Korean J Parasitol. 2015;53(3):299-305.

8. Song EJ, Sohn YM, Ryu KN, Min SY, Shin SH, Park YK. Breast sparganosis and incidentally detected subcutaneous and intramuscular sparganosis at several sites: case report and literature review. Jpn J Radiol. 2015:33(4):225-8.

9. Wiwanitkit V. A review of human sparganosis in Thailand. Int J Infect Dis [Internet]. 2005 Nov [cited 2014 Dec 15]:9(6):312-6. Available from: http:// www.ncbi.nlm.nih.gov/pubmed/16023879

10. Lee SH, Chai J-Y, Seo BS, Cho SY. Two cases of human infection by adult of Spirometra erinacei. Korean J Parasitol. 1984;22(1):66-71.
11. Wang $H$, Tang $Y$, Yang $Y$. A case of Spirometra mansoni infection with both plerocercoid larvae and adult worm. Chinese J Parasitol Parasit Dis. 2012;30(1):40

12. Liu D, Chen J, Fang W. Spirometra. In: Liu D, editor. Molecular detection of human parasitic pathogens. CRC press; 2013. p. 287-95.

13. Guerrant R, Walker D, Weller P. Tropical infectious diseases: principles, pathogens and practice. 3rd Editio. In: Saunders; 2011.

14. Conlan J, Sripa B, Attwood S. Newton P. A review of parasitic zoonoses in a changing Southeast Asia et Parasitol. 2011;182(1):22-40.

15. Mulle R, Wakelin D. Worms and Human Disease. 2nd Editio. Environmental Sciences. CABI publishing; 2002.

16. Vortel V, Pur J, Halberstadt P, Valkounová J. [Human sparganosis] [article in Czech]. Ces Patol. 1995:31(1):3-8.

\section{Submit your next manuscript to BioMed Central and we will help you at every step:}

- We accept pre-submission inquiries

- Our selector tool helps you to find the most relevant journal

- We provide round the clock customer support

- Convenient online submission

- Thorough peer review

- Inclusion in PubMed and all major indexing services

- Maximum visibility for your research

Submit your manuscript at www.biomedcentral.com/submit
Biomed Central 